\title{
On Hartman almost periodic functions
}

\author{
by \\ Guy Cohen (Jerusalem) and Viktor Losert (Wien)
}

\begin{abstract}
We consider multi-dimensional Hartman almost periodic functions and sequences, defined with respect to different averaging sequences of subsets in $\mathbb{R}^{d}$ or $\mathbb{Z}^{d}$. We consider the behavior of their Fourier-Bohr coefficients and their spectrum, depending on the particular averaging sequence, and we demonstrate this dependence by several examples. Extensions to compactly generated, locally compact, abelian groups are considered. We define generalized Marcinkiewicz spaces based upon arbitrary measure spaces and general averaging sequences of subsets. We extend results of Urbanik to locally compact abelian groups.
\end{abstract}

1. Introduction. Let $f$ be a locally integrable function on $\mathbb{R}^{+}$, and assume that the limit

$$
\sigma_{f}(\lambda)=\lim _{T \rightarrow \infty} \frac{1}{T} \int_{0}^{T} f(t) e^{-i \lambda t} d t
$$

exists for every $\lambda \in \mathbb{R}$. Following Kahane [9], such functions are called Hartman almost periodic. $\left(\sigma_{f}(\lambda): \lambda \in \mathbb{R}\right)$ is called the family of FourierBohr coefficients of $f$, and the set $\left\{\lambda: \sigma_{f}(\lambda) \neq 0\right\}$ is called the spectrum of $f$. Answering a question of Hartman (see [6] for further exposition and motivations), J.-P. Kahane proved in [8] that the spectrum is countable.

Similar statements are true in the discrete case. More precisely, let $\left(a_{n}\right)$ be a complex sequence for which the limit

$$
\sigma_{a}(\lambda)=\lim _{N \rightarrow \infty} \frac{1}{N} \sum_{n=1}^{N} a_{n} e^{-i \lambda n}
$$

2000 Mathematics Subject Classification: Primary 42B35; Secondary 11K70, 42A75, $43 \mathrm{~A} 60$.

Key words and phrases: Fourier-Bohr coefficients, Hartman almost periodic functions, generalized Besicovitch spaces, generalized Marcinkiewicz spaces, Følner sequences, generalized means.

This work was done while the first author was staying at the Erwin Schrödinger Institute (ESI), Vienna, Austria, supported by the FWF Project P16004-N05. 
exists for every $\lambda \in[-\pi, \pi]$ (such a sequence is called Hartman almost periodic in $[10$, p. 72$])$. Then the spectrum of $\left(a_{n}\right)$, i.e., $\left\{\lambda: \sigma_{a}(\lambda) \neq 0\right\}$, is countable.

An easy method to generate Hartman almost periodic sequences is as follows. Let $\theta$ be a measure preserving transformation on some probability space and take $g \in L^{1}$. For each $\omega$ in the space define the sequence $a_{n}(\omega)=$ $g\left(\theta^{n} \omega\right)$. The Wiener-Wintner theorem [20] shows that for almost every $\omega$ the sequence $\left(a_{n}(\omega)\right)$ is Hartman almost periodic.

More recently, the study of Hartman almost periodic sequences was motivated by weighted ergodic theorems (pointwise and in norm). Many authors, like Tempelman [18], Ryll-Nardzewski [17], Bellow and Losert [2], Lin, Olsen and Tempelman [12], Çömez, Lin and Olsen [5], Lin and Tempelman [13], Berend et al. [3], have developed various results in this direction. Some of these results ([18], [13], and [3]) extend to actions of locally compact abelian groups, and in some cases, identification of the limit is possible by using the Fourier-Bohr coefficients of the sequence. The description of the limit is considerably simplified because the spectrum is countable.

One of the aims of our paper is to investigate properties of multi-dimensional Hartman almost periodic functions and sequences. In Sec. 4 we consider two-dimensional generalizations of Hartman almost periodic functions and sequences. We give a generalization of Kahane's [8, Theorem 1] when the Fourier-Bohr coefficients are defined by unrestricted convergence, using averages over the family of subsets $[0, S] \times[0, T]$ in $\mathbb{R}^{2}$, with $S, T>0$ (ordered by inclusion). It is shown that for any locally integrable function $f$ the set of $\boldsymbol{\lambda} \in \mathbb{R}^{2}$ for which $\sigma_{f}(\boldsymbol{\lambda})$ exists and is non-zero can be at most countable. If $f$ is Hartman almost periodic, the set $\left\{\boldsymbol{\lambda} \in \mathbb{R}^{2}:\left|\sigma_{f}(\boldsymbol{\lambda})\right| \geq \varepsilon\right\}$ is scattered for every $\varepsilon>0$. The proof uses some slight extensions of the method of Kahane. We also consider a corresponding result for Hartman almost periodic sequences, and discuss various generalizations, in particular to functions defined on compactly generated, locally compact, abelian groups.

On the other hand, for Fourier-Bohr coefficients obtained by considering convergence along squares (in $\mathbb{Z}^{2}$ ), we give an example of a Hartman almost periodic function in this weaker sense (even belonging to $\mathcal{M}_{1}$ - see below) with uncountable spectrum.

In Sec. 5 we discuss related results (positive and negative) in the onedimensional case, concerning convergence along subsequences.

In some sense, Hartman almost periodicity is a minimal requirement for reasonable weights in ergodic theorems. But most of the results in the papers mentioned above need additional growth or regularity properties. Marcinkiewicz [15] has investigated the "Besicovitch spaces" which for 
$1 \leq p<\infty$ consist of locally integrable functions on $\mathbb{R}$ satisfying

$$
\|f\|_{p}:=\limsup _{T \rightarrow \infty}\left(\frac{1}{2 T} \int_{-T}^{T}|f(t)|^{p} d t\right)^{1 / p}<\infty .
$$

He proved that for every $1 \leq p<\infty$ the corresponding space is complete. These are now called Marcinkiewicz spaces and we denote them by $\mathcal{M}_{p}$. In Sec. 2, we define generalized Marcinkiewicz spaces based upon arbitrary measure spaces and limits of averages over more general families of sets. We give a sufficient condition for completeness, and conclude that section by several examples for which our sufficient condition holds. We also give an example of a non-complete space.

Then in Sec. 3 we consider the case of a non-compact, locally compact, abelian group $G$ with Haar measure and a family of sets of "Følner's type". We denote by $\mathbf{B}_{p}$ the space of (generalized) p-Besicovitch almost periodic functions (or sequences), i.e., the closure in $\mathcal{M}_{p}$ of the continuous almost periodic functions (sequences) on $G$.

Urbanik [19] has shown (for $\mathbb{R}$ ) that if a Hartman almost periodic function $f$ is in $\mathcal{M}_{p}$ for some $p>1$, then $\left(\sigma_{f}(\lambda): \lambda \in \mathbb{R}\right)$ is the family of Fourier-Bohr coefficients of a Besicovitch almost periodic function from $\mathbf{B}_{p}$. Equivalently, it is the family of (the usual) Fourier coefficients of a function $h^{*} \in L_{p}\left(\mathbb{R}^{*}\right)$, where $\mathbb{R}^{*}$ denotes the Bohr compactification of $\mathbb{R}$. Here the definition of $\sigma_{f}(\lambda)$ can be extended by using "generalized mean values" (which covers, e.g., all kinds of subsequential convergence). This can be generalized to arbitrary $G$ (without much difficulty).

\section{Generalized Marcinkiewicz spaces}

Definition 2.1. Let $(\Omega, \mu)$ be an infinite measure space, and let $\left(\Omega_{i}: i \in I\right)$ be a family of measurable subsets in $\Omega$ of finite measure, where $I$ is some directed index set. Let $f$ be a measurable complex-valued function on $\Omega$, and for $1 \leq p<\infty$ define

$$
\|f\|_{p}=\limsup _{i \in I}\left(\frac{1}{\mu\left(\Omega_{i}\right)} \int_{\Omega_{i}}|f(x)|^{p} \mu(d x)\right)^{1 / p} .
$$

We call $\mathcal{M}_{p}:=\left\{f:\|f\|_{p}<\infty\right\}$ a generalized Marcinkiewicz space (with respect to the family $\left(\Omega_{i}\right)$ ).

By elementary arguments, $\mathcal{M}_{p}$ is a linear space, $\|\cdot\|_{p}$ is a seminorm on $\mathcal{M}_{p}$. Furthermore, we have $\|\cdot\|_{p} \leq\|\cdot\|_{p^{\prime}}$ and in particular $\mathcal{M}_{p^{\prime}} \subseteq \mathcal{M}_{p}$ for $p \leq p^{\prime}$. For $1 / p+1 / q=1$, we have $\|f g\|_{1} \leq\|f\|_{p}\|g\|_{q}$, hence $\mathcal{M}_{p} \cdot \mathcal{M}_{q} \subseteq \mathcal{M}_{1}$.

The question of completeness of the Marcinkiewicz spaces has turned out to be somewhat subtle. We give some partial results. 
Proposition 2.2. Assume that for some $1 \leq p<\infty$, the family $\left(\Omega_{i}: i \in I\right)$ of measurable subsets of $\Omega$ satisfies the following condition:

$(\mathcal{M}) \quad$ For any $f$ with $\|f\|_{p}>0$ there exists $f^{*}$ such that

$$
\left\|f-f^{*}\right\|_{p}=0, \quad \sup _{i \in I}\left(\frac{1}{\mu\left(\Omega_{i}\right)} \int_{\Omega_{i}}\left|f^{*}(x)\right|^{p} \mu(d x)\right)^{1 / p} \leq 2\|f\|_{p} .
$$

Then the Marcinkiewicz space $\mathcal{M}_{p}$ is complete.

Proof. A well known characterization says that a normed (or seminormed) space is complete iff every absolutely converging series converges (i.e., convergence of $\sum\left\|v_{n}\right\|$ implies convergence of $\sum v_{n}$ ). Assume that $\sum_{n}\left\|f_{n}\right\|_{p}$ converges. By property $(\mathcal{M})$, there exists a sequence $\left\{f_{n}^{*}\right\}$ such that $\left\|f_{n}-f_{n}^{*}\right\|_{p}=0$ and for every $n \geq 1$,

$$
\sup _{i \in I}\left(\frac{1}{\mu\left(\Omega_{i}\right)} \int_{\Omega_{i}}\left|f_{n}^{*}(x)\right|^{p} \mu(d x)\right)^{1 / p} \leq 2\left\|f_{n}\right\|_{p} .
$$

Clearly, norm convergence of $\sum_{n} f_{n}^{*}$ is equivalent to norm convergence of $\sum_{n} f_{n}$ (to the same limit), so it is enough to show convergence of $\sum_{n} f_{n}^{*}$. For each $\omega \in \Omega$, define $f(\omega)=\sum_{n} f_{n}^{*}(\omega)$ if the series converges, otherwise put $f(\omega)=0$. By property $(\mathcal{M})$ and by the Beppo Levi theorem, for a.e. $\omega \in \Omega_{i}$ the series $\sum_{n} f_{n}^{*}(\omega)$ is absolutely convergent. It easily follows that $\left\|\sum_{k=1}^{n} f_{k}^{*}-f\right\|_{p} \rightarrow_{n} 0$.

We will now give several natural examples where property $(\mathcal{M})$ is satisfied.

EXAmPLes 2.3. (i) Let $I=\mathbb{N}$ (with natural order) and let $\left(\Omega_{n}\right)$ be any (not necessarily increasing) sequence of measurable subsets satisfying $\mu\left(\Omega_{n}\right) \rightarrow \infty$. For $f \in \mathcal{M}_{p}$, let $n_{0}>0$ be an integer for which

$$
\sup _{n>n_{0}}\left(\frac{1}{\mu\left(\Omega_{n}\right)} \int_{\Omega_{n}}|f(x)|^{p} \mu(d x)\right)^{1 / p} \leq 2\|f\|_{p} .
$$

Define $f^{*}$ as follows: $f^{*}(x)=0$ if $x \in \bigcup_{n \leq n_{0}} \Omega_{n}$, otherwise put $f^{*}(x)=f(x)$.

A specific example is the following: Let $\left(b_{i} \geq 1\right)\left(n_{i} \geq 0\right)$ be sequences of integers, and assume that $b_{i} \rightarrow \infty$. Let $\Omega=\mathbb{N}, \Omega_{i}=\left\{n_{i}+1, \ldots, n_{i}+b_{i}\right\}$, and let $\mu$ be the counting measure. The authors of [1] (and others) considered "moving averages" of the form $\left(1 / b_{i}\right) \sum_{k=n_{i}+1}^{n_{i}+b_{i}} a_{k}$, giving rise to subsequential ergodic theorems. Now, we see that the corresponding generalized Marcinkiewicz spaces are complete.

(ii) Given an arbitrary measure space, assume that we have a subset $I_{0}$ of $I, i_{1} \in I$ and $c>0$ such that for any $i \in I$ with $i \geq i_{1}$ there exists 
$i_{0} \in I_{0}$ with $i \leq i_{0}, \Omega_{i} \subseteq \Omega_{i_{0}}$ and $\mu\left(\Omega_{i}\right) \geq c \mu\left(\Omega_{i_{0}}\right)$ (i.e., $I_{0}$ is cofinal and "sufficiently dense" in some tail of $I)$. Then $\left(\Omega_{i}: i \in I\right)$ and $\left(\Omega_{i}: i \in I_{0}\right)$ define the same Marcinkiewicz spaces $\mathcal{M}_{p}$ (with equivalent seminorms $\|\cdot\|_{p}$ ). Furthermore, if $I_{0}$ is countable and linearly ordered and $\mu\left(\Omega_{i}\right) \rightarrow \infty$, then (compare (i)) property $(\mathcal{M})$ holds for $\left(\Omega_{i}: i \in I_{0}\right)$ (essentially, it holds also for $\left(\Omega_{i}: i \in I, i \geq i_{1}\right)$, but possibly with a different constant), in particular the spaces $\mathcal{M}_{p}$ are complete.

This covers the classical example of Marcinkiewicz [15], where $\Omega=\mathbb{R}$ and $\Omega_{t}=[-t, t]$. Similarly for $\Omega_{t}=[0, t]$.

(iii) Another example arises from the setting of Theorem 4.3 below, where $\Omega=\mathbb{R}^{2}$ with standard Lebesgue measure and one considers the family of all rectangles $[0, S] \times[0, T]$ with $S, T>0$ (ordered by inclusion). In this case the family is not linearly ordered.

(iv) Let $\Omega=\mathbb{R}$ (with standard Lebesgue measure) with the family of all intervals $[a, b]$ where $a<b$. It is easy to see that property $(\mathcal{M})$ does not hold, but again (ii) applies. Passing to the subfamily defined by any subset $I_{1}=\left\{i \in I: i \geq i_{1}\right\}$ it follows that this gives the same Marcinkiewicz spaces $\mathcal{M}_{p}$ as the classical family $\Omega_{t}=[-t, t]$.

In [4, Ch. I] Marcinkiewicz spaces have been investigated for $\Omega=\mathbb{R}^{d}$ (with standard Lebesgue measure) based on the family $\Omega_{n}=n \Gamma(n \in \mathbb{N})$, where $\Gamma$ is some bounded convex neighborhood of the origin (to prove completeness in this case, the authors of [4] already used the same technique as in the proof of Proposition 2.2 above). By (ii), these spaces do not depend on the choice of $\Gamma$ (of course the seminorms will depend in general) and one can take for $\Gamma$ any bounded measurable neighborhood of the origin and also use the family of translates $\left\{\mathbf{x}+n \Gamma: \mathbf{x} \in \mathbb{R}^{d}, n \in \mathbb{N}\right\}$ (ordered by inclusion). But for $d>1$, different spaces are obtained when using the family of all bounded convex subsets with non-empty interior, and for $d=2$ these are also different from those obtained from the family of rectangles $[-S, S] \times[-T, T]$ with $S, T>0$ (related to the notion of unrestricted convergence considered in Section 4).

(v) Let $G$ be a compactly generated locally compact group, and $\mu$ Haar measure. If $U$ is a symmetric, relatively compact, measurable neighborhood of the identity, generating $G$ (i.e., $G=\bigcup_{n=1}^{\infty} U^{n}$ ), it is well known that there exists $d_{0}>0$ such that $\mu\left(U^{n+1}\right) \leq d_{0} \mu\left(U^{n}\right)$ for all $n$. It follows that the Marcinkiewicz spaces $\mathcal{M}_{p}$ defined by the family $\left(U^{n}: n \in \mathbb{N}\right)$ are complete and do not depend on the choice of $U$.

It seems unlikely that property $(\mathcal{M})$ and variations like (ii) cover all the cases where $\mathcal{M}_{p}$ is complete, but we want to give also an example where the Marcinkiewicz space $\mathcal{M}_{p}$ is not complete: 
EXAmple 2.4. Take $\Omega=\mathbb{N}$ (with counting measure) and consider the family of all sets $A \cup B$ where $A, B$ are bounded subintervals of $\mathbb{N}$ (ordered by inclusion). Then the following properties can be verified easily: any $f \in \mathcal{M}_{p}$ is bounded, and in particular all the spaces $\mathcal{M}_{p}$ coincide in this case for $1 \leq p<\infty$. Define $f_{n}$ by $f_{n}(k)=2^{n} / n^{2}$ if $2^{n} \mid k$ and $f_{n}(k)=0$ otherwise $(n=1,2, \ldots)$; then $\left\|f_{n}\right\|_{1} \leq 3 / n^{2}$, but $\sum_{n=1}^{\infty} f_{n}$, which exists pointwise, does not converge in $\mathcal{M}_{1}$ (since it represents an unbounded sequence). Thus the spaces $\mathcal{M}_{p}$ are not complete in this case.

Remark. Let $\mathbf{X}$ be a separable Banach space with norm $\|\cdot\|_{\mathbf{X}}$. Let $f$ be an $\mathbf{X}$-valued measurable function, defined on $\Omega$. For such functions, one can consider a seminorm $\|f\|_{p}:=\|\| f\left\|_{\mathbf{X}}\right\|_{p}$. Proposition 2.2 remains true and the generalized Marcinkiewicz spaces $\mathcal{M}_{p}(\mathbf{X}):=\left\{f:\|f\|_{p}<\infty\right\}$ are complete if condition $(\mathcal{M})$ holds.

3. The Besicovitch spaces $\mathbf{B}_{p}$. Let $G$ be a non-compact locally compact group with Haar measure $\mu$, let $I$ be a directed set and let $\left(A_{i}: i \in I\right)$ be a family of measurable subsets of $G$, satisfying two conditions of " $\mathrm{F} \varnothing$ lner's type"

(i) $0<\mu\left(A_{i}\right)<\infty$ for all $i \in I$,

(ii) $\lim _{i \in I} \mu\left(x A_{i} \triangle A_{i}\right) / \mu\left(A_{i}\right)=0$ for all $x \in G$.

This is sometimes called an asymptotically left invariant net (of subsets); see [16, p. 48]. Such a net exists if and only if the group $G$ is amenable.

Let $\operatorname{AP}(G)$ be the space of continuous almost periodic functions on $G$ and let $G^{*}$ be the Bohr compactification of $G([16$, p. 284]). There is an isometric isomorphism (denoted as $g \mapsto g^{*}$ ) between $\operatorname{AP}(G)$ and the space of continuous functions $C\left(G^{*}\right)$ (with supremum norm $\|\cdot\|_{\infty}$ ).

For $1 \leq p<\infty$, we consider $\mathcal{M}_{p}$, the generalized Marcinkiewicz spaces as in Definition 2.1, with respect to such a family $\left(A_{i}: i \in I\right)$. We denote by $\mathbf{B}_{p}$ the closure of $\operatorname{AP}(G)$ in $\mathcal{M}_{p}$ with respect to the seminorm $\|\cdot\|_{p}$. We call $\mathbf{B}_{p}$ the space of Besicovitch almost periodic functions on $G$ (see also [11] for a related definition). Clearly, $\mathbf{B}_{p^{\prime}} \subseteq \mathbf{B}_{p}$ for $p \leq p^{\prime}$ and $\mathbf{B}_{p} \cdot \operatorname{AP}(G) \subseteq \mathbf{B}_{p}$.

Let $\mu^{*}$ be the normalized Haar measure on $G^{*}$. On the space $\operatorname{AP}(G)$ there is a unique two-sided translation invariant mean $m$ and it satisfies

$$
m(g)=\int_{G^{*}} g^{*}(x) \mu^{*}(d x) .
$$

In addition, uniqueness implies the formula

$$
m(g)=\lim _{i \in I} \frac{1}{\mu\left(A_{i}\right)} \int_{A_{i}} g(x) \mu(d x) \quad \text { for every } g \in \mathrm{AP}(G)
$$

(see, e.g., [16, Proposition 22.21]). 
In particular, $\|g\|_{p}=\left\|g^{*}\right\|_{p}$ for $g \in \operatorname{AP}(G)$ (on the right side we consider the $L^{p}\left(G^{*}, \mu^{*}\right)$-norm). It follows that the correspondence $g \mapsto g^{*}$ extends to an isometric mapping (denoted in the same way) of $\mathbf{B}_{p}$ into $L_{p}\left(G^{*}, \mu^{*}\right)$. Furthermore, the mean $m$ has a unique continuous extension to the whole space $\mathbf{B}_{1}$. By the Hahn-Banach extension theorem the functional $m$ can be further extended to a linear functional on $\mathcal{M}_{1}$, without increasing its norm. Following Urbanik [19], such an extension will be called a generalized mean on $\mathcal{M}_{1}$. In general, there are many extensions and any such extension will be still denoted by $m$.

For the next two results, we restrict to the case where $G$ is abelian. (Similar statements hold in the general case, when considering continuous finite-dimensional representations instead of characters. But observe that there are some non-abelian groups, e.g., $G=\mathrm{SL}(2, \mathbb{R})$, for which $\operatorname{AP}(G)$ contains just the constant functions - see [7, 22.22].) Let $\widehat{G}$ be the dual group of $G$. The Bohr compactification $G^{*}$ can be identified with the group of (not necessarily continuous) characters of $G$. Algebraically, $\widehat{G}$ is isomorphic to the dual group of $G^{*}$. Continuous characters on $G$ will be denoted by $\chi$. We consider $G$ as a dense subgroup of $G^{*}$. (But the topology of $G$ is in general strictly finer than the induced topology.) Then $\chi^{*}$ is just the unique extension of $\chi$ to a continuous character on $G^{*}$. Conversely, the restriction to $G$ of a continuous character on $G^{*}$ is a continuous character on $G$ ( $[7$, Theorem 26.12]). Therefore we use the same letters for continuous characters on $G$ and those on $G^{*}$.

For a function $g \in \mathbf{B}_{1}$, we have $g \chi \in \mathbf{B}_{1}$ for any $\chi \in \widehat{G}$. The value $m(g \bar{\chi})$ is called the Fourier-Bohr coefficient of $g$ at $\chi$; it is exactly the (usual) Fourier coefficient $\widehat{g}^{*}(\chi)=\int_{G^{*}} g^{*} \bar{\chi} d \mu$, using again the extension of $\chi$ to a character of $G^{*}$ (we follow here the habits of abelian harmonic analysis, e.g. as in [19], [7]; they are different from the definition of Fourier transforms common in the non-abelian case). The spectrum of $g$ is the set $\{\chi \in \widehat{G}: m(g \bar{\chi}) \neq 0\}$. Since for every $\varepsilon>0$ the set $\left\{\chi \in \widehat{G}:\left|\widehat{g^{*}}(\chi)\right| \geq \varepsilon\right\}$ is finite, every Besicovitch almost periodic function $\left(g \in \mathbf{B}_{1}\right)$ has a countable spectrum.

The following two results are generalizations of Theorem 3 and Corollary 2 of Urbanik [19].

THEOREM 3.1. Let $m$ be a generalized mean on $\mathcal{M}_{1}$. If $1<p<\infty$, then for any $f \in \mathcal{M}_{p}$, there exists $f_{1} \in L_{p}\left(G^{*}, \mu^{*}\right)$ such that $m(f \bar{\chi})=\widehat{f}_{1}(\chi)$ for every $\chi \in \widehat{G}$. It follows that for every $\varepsilon>0$ the set $\{\chi \in \widehat{G}:|m(f \bar{\chi})| \geq \varepsilon\}$ is finite, in particular, $m(f \bar{\chi})=0$ for all $\chi \in \widehat{G}$ outside a countable subset.

Proof. Fix $f \in \mathcal{M}_{p}$ and let $1<q<\infty$ be the dual index of $p>1$. We define a linear functional $l$ on $C\left(G^{*}\right)$ by $l\left(g^{*}\right)=m(f g)$. 
Clearly

$$
\left|l\left(g^{*}\right)\right|=|m(f g)| \leq\|f g\|_{1} \leq\|f\|_{p}\|g\|_{q}=\|f\|_{p}\left\|g^{*}\right\|_{q},
$$

hence $l$ extends to a continuous functional on $L_{q}\left(G^{*}, \mu^{*}\right)$. Thus, there exists a function $f_{1} \in L_{p}\left(G^{*}, \mu^{*}\right)$ such that for any $g^{*} \in L_{q}\left(G^{*}, \mu^{*}\right)$ we have

$$
l\left(g^{*}\right)=\int_{G^{*}} f_{1} g^{*} d \mu^{*} .
$$

Hence, $m(f \bar{\chi})=l(\bar{\chi})=\widehat{f}_{1}(\chi)$. All the assertions on $\{m(f \bar{\chi}): \chi \in \widehat{G}\}$ follow from the fact that it is the set of Fourier coefficients of some function from $L_{p}\left(G^{*}, \mu^{*}\right)$.

For $\chi \in \widehat{G}$, we put

$$
\sigma_{f}(\chi)=\lim _{i \in I} \frac{1}{\mu\left(A_{i}\right)} \int_{A_{i}} f \chi d \mu,
$$

whenever the limit exists.

Corollary 3.2. Let $f$ be a function in $\mathcal{M}_{p}, 1<p<\infty$, and put

$$
\underline{\sigma}_{f}(\chi)=\liminf _{i \in I}\left|\frac{1}{\mu\left(A_{i}\right)} \int_{A_{i}} f \bar{\chi} d \mu\right| .
$$

Then for every $\varepsilon>0$, the set $\left\{\chi \in \widehat{G}: \underline{\sigma}_{f}(\chi) \geq \varepsilon\right\}$ is finite. In particular, the set where $\underline{\sigma}_{f}(\chi) \neq 0$ can be at most countable.

Proof. Considering a universal refinement of the net, we can assume that

$$
m(h):=\lim _{i \in I} \frac{1}{\mu\left(A_{i}\right)} \int_{A_{i}} h d \mu
$$

exists for all $h \in \mathcal{M}_{1}$. Clearly, $m$ is a generalized mean, $|m(f \bar{\chi})| \geq \underline{\sigma}_{f}(\chi)$ and $m(f \bar{\chi})=\sigma_{f}(\chi)$ (when the original limit exists). So, the previous theorem yields the result.

Remarks. 1. Theorem 3.1 and Corollary 3.2 were proved in Urbanik [19] more generally for Orlicz space type Marcinkiewicz spaces on $\mathbb{R}$. One can prove the above results similarly for generalized Orlicz-Marcinkiewicz spaces over $G$.

2. If $G$ is a locally compact, $\sigma$-compact amenable group, there always exists a sequence of measurable subsets $\left(A_{n}\right)$ of $G$, satisfying (i) and (ii) above ([16, Proposition 16.11], these are called "averaging sequences"; see also [7, Theorem 18.13] for the abelian case). For such a sequence, it follows from Proposition 2.2 that the generalized Marcinkiewicz spaces $\mathcal{M}_{p}$ are all complete, hence the same is true for the spaces $\mathbf{B}_{p}$, and $\mathbf{B}_{p}$ is isomorphic to $L_{p}\left(G^{*}, \mu^{*}\right)$. In particular, one can conclude in Theorem 3.1 that $f_{1}=h^{*}$ for some $h \in \mathbf{B}_{p}$. 
Typical examples of applications of Theorem 3.1 and Corollary 3.2 are given by considering $G=\mathbb{R}^{d}$ (or $G=\mathbb{Z}^{d}$ ), $\mu$ the Lebesgue measure (or the counting measure), and $A_{t}=[-t, t]^{d}$ (or $A_{n}=[-n, n]^{d}$ ).

In the $\sigma$-compact case, one can always take $\left(A_{n}\right)$ to be open subsets with compact closure such that $A_{1} \subseteq A_{2} \subseteq \cdots$ and $\bigcup_{n=1}^{\infty} A_{n}=G$ (as above). Observe that if $G$ is any non-compact locally compact group and $\left(A_{n}\right)$ is a sequence of measurable sets satisfying (i) and (ii) above, then necessarily $\mu\left(A_{n}^{-1}\right) \rightarrow \infty$. Indeed, consider

$$
\phi_{n}(x)=\frac{\mu\left(x A_{n} \cap A_{n}\right)}{\mu\left(A_{n}\right)} .
$$

This is a sequence of continuous positive definite functions on $G$ which by (ii) converges pointwise to 1 , hence by Lebesgue's theorem, it converges for the weak*-topology $\sigma\left(L^{\infty}, L^{1}\right)$; but then by a classical theorem of Raikov, we get uniform convergence on compact sets. Thus (ii) holds uniformly for $x$ in a compact subset of $G$. Let $U$ be a compact subset of $G$; then, denoting the indicator functions by $c_{A_{n}}, c_{U}$ and convolution by $*$, it follows that

$$
\frac{1}{\mu\left(A_{n}\right)}\left\|\frac{c_{U}}{\mu(U)} * c_{A_{n}}-c_{A_{n}}\right\|_{1} \rightarrow 0 .
$$

Hence, for $n$ large enough, there exists $x \in A_{n}$ such that $\mu\left(U \cap x A_{n}^{-1}\right)=$ $c_{U} * c_{A_{n}}(x) \geq \frac{1}{2} \mu(U)$, proving our claim.

Of course, if $G$ is unimodular, it follows that $\mu\left(A_{n}\right) \rightarrow \infty$, but if $G$ is not unimodular, one can use right translations to make $\mu\left(A_{n}\right)$ as small or large as one likes. On the other hand, if the underlying discrete group of $G$ is amenable, one can always construct a net satisfying (i), (ii) and $\mu\left(A_{i}\right) \rightarrow 0$.

In the non- $\sigma$-compact case, sequences cannot be sufficient, and presumably the Besicovitch spaces will show a rather pathological behavior.

3. Corollary 3.2 in general fails to hold for $p=1$ (but of course, it stays true when $\left.f \in \mathbf{B}_{1}\right)$. Considering $G=\mathbb{R}$ and the intervals $A_{n}=[-n !, n !]$, Urbanik [19, Theorem 2] has constructed a function $f \in \mathcal{M}_{1}$ such that $\sigma_{f}\left(\chi_{t}\right) \neq 0$ for uncountably many $t$ (where $\chi_{t}(x)=e^{i t x}$; but in this example $\sigma_{f}\left(\chi_{t}\right)$ does not exist for some $\left.t\right)$. The limit of the averages extends (as above) to a generalized mean $m$ on $\mathcal{M}_{1}$, and this also gives a counter-example to Theorem 3.1 when $p=1$.

\section{Hartman almost periodic functions}

Definition 4.1. Let $G$ be a locally compact, abelian group, and let $\mu$ be a Haar measure on $G$. Let $\left(A_{i}: i \in I\right)$ be a family of measurable subsets of $G$, satisfying conditions (i) and (ii) of Section 3, where $I$ is some directed 
index set. A measurable complex-valued function $f$ on $G$ for which

$$
\sigma_{f}(\chi)=\lim _{i \in I} \frac{1}{\mu\left(A_{i}\right)} \int_{A_{i}} f \bar{\chi} d \mu
$$

exists for every $\chi \in \widehat{G}$ will be called a Hartman almost periodic function (this goes back to Kahane: see [9] for $G=\mathbb{R}$ with $A_{t}=[-t, t], t \in \mathbb{R}^{+}$, and $[10$, p. 72$]$ for $G=\mathbb{Z}$ with $\left.A_{k}=[-k, k], k \in \mathbb{N}\right)$. The spectrum of $f$ is the set $\left\{\chi \in \widehat{G}: \sigma_{f}(\chi) \neq 0\right\}$.

As mentioned before, every Besicovitch almost periodic function $\left(f \in \mathbf{B}_{1}\right)$ is Hartman almost periodic and $\sigma_{f}(\chi)$ has the same properties as in Corollary 3.2. Furthermore, if $f$ is Hartman almost periodic and in addition $f \in \mathcal{M}_{p}$ holds for some $p$ with $1<p<\infty$, then Corollary 3.2 applies as well, i.e., in these cases $\left\{\chi \in \widehat{G}:\left|\sigma_{f}(\chi)\right| \geq \varepsilon\right\}$ is finite for every $\varepsilon>0$ (without further assumptions on the family $\left(A_{i}\right)$, and for arbitrary locally compact abelian groups).

In [9] Kahane has shown that there are quite many functions outside $\mathbf{B}_{1}$ that are Hartman almost periodic (in particular, there is no hope to recover $f$ from $\sigma_{f}(\chi)$, in general). Kahane [8, Théorème 1] proved that all Hartman almost periodic functions on $\mathbb{R}$, with $A_{t}=[0, t], t \in \mathbb{R}^{+}$, have countable spectrum. It turns out that such general properties of $\sigma_{f}(\chi)$ depend very much on the choice of the family $\left(A_{i}\right)$ (loosely speaking: if $\left(A_{i}\right)$ fills out the space more regularly, one gets a more restricted class of coefficients $\left.\sigma_{f}(\chi)\right)$. Some examples on this in the one-dimensional case will be given in the Remarks of Section 5.

In this section we concentrate on the case $G=\mathbb{R}^{2}$, looking for generalizations of Kahane's theorem. Results for $\mathbb{R}^{d}, d>2$, can be shown in a similar manner (with some more notational effort). Identifying $\widehat{\mathbb{R}^{2}}$ with $\mathbb{R}^{2}$, we write $\sigma_{f}(\boldsymbol{\lambda})$ instead of $\sigma_{f}\left(\chi_{\boldsymbol{\lambda}}\right)$, where for $\boldsymbol{\lambda}=\left(\lambda^{(1)}, \lambda^{(2)}\right) \in \mathbb{R}^{2}, \chi_{\boldsymbol{\lambda}}(s, t)=$ $e^{i\left(\lambda^{(1)} s+\lambda^{(2)} t\right)}$. Again the choice of $\left(A_{i}\right)$ is essential.

Definition 4.2. Let $h(s, t)$ be a complex-valued function on $\mathbb{R}^{2}$. We say that $L$ is the unrestricted limit of $h(s, t)$ as $s, t \rightarrow \infty$ if for every $\varepsilon>0$ there exists $M>0$ such that for every $s, t \geq M$ we have $|h(s, t)-L| \leq \varepsilon$.

From now on, all the limits considered will be unrestricted limits. In our general notation, this means that we consider Example 2.3(iii), where $I=\mathbb{R}^{+} \times \mathbb{R}^{+}$and $A_{S T}=[0, S] \times[0, T]$. Clearly, we can always restrict to functions on $\mathbb{R}^{+} \times \mathbb{R}^{+}$.

THEOREM 4.3. Let $f(s, t)$ be a locally integrable function on $[0, \infty) \times$ $[0, \infty)$, and let $F \subset \mathbb{R}^{2}$ be closed. Suppose that for each $\boldsymbol{\lambda}=\left(\lambda^{(1)}, \lambda^{(2)}\right) \in F$ 
the limit

$$
\sigma_{f}(\boldsymbol{\lambda}):=\lim _{S, T \rightarrow \infty} \frac{1}{S T} \int_{0}^{S} \int_{0}^{T} f(s, t) e^{-i\left(\lambda^{(1)} s+\lambda^{(2)} t\right)} d s d t
$$

exists. Then $\sigma_{f}(\boldsymbol{\lambda})=0$ for all $\boldsymbol{\lambda} \in F$ outside a countable subset. Furthermore, given $\varepsilon>0$, the set $F_{\varepsilon}:=\left\{\boldsymbol{\lambda} \in F:\left|\sigma_{f}(\boldsymbol{\lambda})\right| \geq \varepsilon\right\}$ does not contain any subset which is dense in itself, i.e., $F_{\varepsilon}$ is a scattered set.

Proof. Recall that a scattered subset of $\mathbb{R}^{2}$ is countable (since the plane satisfies the second-countability axiom), thus it will be enough to show the second statement.

For every $\boldsymbol{\lambda} \in F$ define

$$
\sigma_{f}(\boldsymbol{\lambda}, S, T):=\frac{1}{S T} \int_{0}^{S} \int_{0}^{T} f(s, t) e^{-i\left(\lambda^{(1)} s+\lambda^{(2)} t\right)} d s d t .
$$

More generally, we consider

$$
\begin{aligned}
\sigma_{f}\left(\boldsymbol{\lambda}, S, T, S_{0}, T_{0}\right) & :=\frac{1}{S T} \int_{S_{0}}^{S} \int_{T_{0}}^{T} f(s, t) e^{-i\left(\lambda^{(1)} s+\lambda^{(2)} t\right)} d s d t \\
\varepsilon_{\boldsymbol{\lambda}}\left(S, T, S_{0}, T_{0}\right) & :=\sigma_{f}\left(\boldsymbol{\lambda}, S, T, S_{0}, T_{0}\right)-\sigma_{f}(\boldsymbol{\lambda}) \\
\bar{\sigma}_{f}\left(\boldsymbol{\lambda}, S_{0}, T_{0}\right) & :=\sup _{S>S_{0}}\left|\sigma_{f}\left(\boldsymbol{\lambda}, S, T_{0}\right)\right|+\sup _{T>T_{0}}\left|\sigma_{f}\left(\boldsymbol{\lambda}, S_{0}, T\right)\right| .
\end{aligned}
$$

Observe that convergence of $\sigma_{f}(\boldsymbol{\lambda}, S, T)$ implies that $\bar{\sigma}_{f}\left(\boldsymbol{\lambda}, S_{0}, T_{0}\right)$ is finite as soon as $S_{0}, T_{0}$ are sufficiently large (the starting point for finiteness may depend on $\boldsymbol{\lambda}$ ). Furthermore, an easy computation shows that

$$
\begin{aligned}
\sigma_{f}\left(\boldsymbol{\lambda}, S, T, S_{0}, T_{0}\right)= & \sigma_{f}(\boldsymbol{\lambda}, S, T)-\frac{S_{0}}{S} \sigma_{f}\left(\boldsymbol{\lambda}, S_{0}, T\right) \\
& -\frac{T_{0}}{T} \sigma_{f}\left(\boldsymbol{\lambda}, S, T_{0}\right)+\frac{S_{0} T_{0}}{S T} \sigma_{f}\left(\boldsymbol{\lambda}, S_{0}, T_{0}\right) .
\end{aligned}
$$

Consequently, $\bar{\sigma}_{f}\left(\boldsymbol{\lambda}, S_{0}, T_{0}\right)<\infty$ implies $\sigma_{f}(\boldsymbol{\lambda})=\lim _{S, T \rightarrow \infty} \sigma_{f}\left(\boldsymbol{\lambda}, S, T, S_{0}, T_{0}\right)$, i.e., $\lim _{S, T \rightarrow \infty} \varepsilon_{\boldsymbol{\lambda}}\left(S, T, S_{0}, T_{0}\right)=0$ (and it is not hard to see that the condition is necessary as well to be able to drop the "initial segment"; see also Remark 1 below).

Our next aim will be (see $(* *)$ below) to derive an asymptotic expression for $\sigma_{f}\left(\boldsymbol{\lambda}+\boldsymbol{\mu}, S, T, S_{0}, T_{0}\right)$ when $\boldsymbol{\lambda}, S_{0}, T_{0}$ are fixed. First, we assume that $f$ is continuous. For brevity, we write $\varepsilon_{\boldsymbol{\lambda}}(S, T)$ instead of $\varepsilon_{\boldsymbol{\lambda}}\left(S, T, S_{0}, T_{0}\right)$ and $e^{\boldsymbol{\lambda}}(s, t)=e^{-i\left(\lambda^{(1)} s+\lambda^{(2)} t\right)}$.

Since $\operatorname{st\varepsilon }_{\boldsymbol{\lambda}}(s, t)=\int_{S_{0}}^{s} \int_{T_{0}}^{t} f(u, v) e^{\boldsymbol{\lambda}}(u, v) d u d v-s t \sigma_{f}(\boldsymbol{\lambda})$, we obtain by Fubini's theorem

$$
\frac{\partial^{2}\left(s t \varepsilon_{\boldsymbol{\lambda}}(s, t)\right)}{\partial s \partial t}=\frac{\partial^{2}\left(s t \varepsilon_{\boldsymbol{\lambda}}(s, t)\right)}{\partial t \partial s}=f(s, t) e^{\boldsymbol{\lambda}}(s, t)-\sigma_{f}(\boldsymbol{\lambda}) .
$$


Fix $\boldsymbol{\lambda} \in F$ and take any $\boldsymbol{\mu}=\left(\mu^{(1)}, \mu^{(2)}\right) \in \mathbb{R}^{2}$. We have

$$
\begin{aligned}
& \sigma_{f}\left(\boldsymbol{\lambda}+\boldsymbol{\mu}, S, T, S_{0}, T_{0}\right) \\
& \quad=\frac{\sigma_{f}(\boldsymbol{\lambda})}{S T} \int_{S_{0}}^{S} \int_{T_{0}}^{T} e^{\boldsymbol{\mu}}(s, t) d s d t+\frac{1}{S T} \int_{S_{0}}^{S} \int_{T_{0}}^{T} \frac{\partial^{2} s t \varepsilon_{\boldsymbol{\lambda}}(s, t)}{\partial s \partial t} e^{\boldsymbol{\mu}}(s, t) d s d t .
\end{aligned}
$$

We denote the second integral of $(*)$ by $I$ and we use integration by parts in order to bring it to a convenient form:

$$
\begin{aligned}
S T I= & \int_{S_{0}}^{S}\left[\int_{T_{0}}^{T} \frac{\partial}{\partial t} \frac{\partial\left(s t \varepsilon_{\boldsymbol{\lambda}}(s, t)\right)}{\partial s} e^{\boldsymbol{\mu}}(s, t) d t\right] d s \\
= & \int_{S_{0}}^{S}\left[\left.\frac{\partial\left(s t \varepsilon_{\boldsymbol{\lambda}}(s, t)\right)}{\partial s} e^{\boldsymbol{\mu}}(s, t)\right|_{t=T_{0}} ^{T}\right] d s \\
& +i \mu^{(2)} \int_{T_{0}}^{T}\left[\int_{S_{0}}^{S} \frac{\partial\left(s t \varepsilon_{\boldsymbol{\lambda}}(s, t)\right)}{\partial s} e^{\boldsymbol{\mu}}(s, t) d s\right] d t .
\end{aligned}
$$

By the definition of partial differentiation, for every $t_{0}$ we have

$$
\left.\frac{\partial\left(s t \varepsilon_{\boldsymbol{\lambda}}(s, t)\right)}{\partial s}\right|_{t=t_{0}}=\frac{\partial\left(s t_{0} \varepsilon_{\boldsymbol{\lambda}}\left(s, t_{0}\right)\right)}{\partial s} .
$$

Considering this equality and using another integration by parts for the second integral above, we obtain

$$
\begin{aligned}
S T I= & \int_{S_{0}}^{S} \frac{\partial\left(s T \varepsilon_{\boldsymbol{\lambda}}(s, T)\right)}{\partial s} e^{\boldsymbol{\mu}}(s, T) d s+i \mu^{(2)} \int_{T_{0}}^{T}\left[\left.s \varepsilon_{\boldsymbol{\lambda}}(s, t) e^{\boldsymbol{\mu}}(s, t)\right|_{s=S_{0}} ^{S}\right] t d t \\
& -\mu^{(1)} \mu^{(2)} \int_{S_{0} T_{0}}^{S} s t \varepsilon_{\boldsymbol{\lambda}}(s, t) e^{\boldsymbol{\mu}}(s, t) d t d s .
\end{aligned}
$$

Another integration by parts for the first integral above yields

$$
\begin{aligned}
I= & \left(\varepsilon_{\boldsymbol{\lambda}}(S, T) e^{\boldsymbol{\mu}}(S, T)-\frac{S_{0}}{S} \varepsilon_{\boldsymbol{\lambda}}\left(S_{0}, T\right) e^{\boldsymbol{\mu}}\left(S_{0}, T\right)\right) \\
& +\frac{i \mu^{(1)}}{S} \int_{S_{0}}^{S} s \varepsilon_{\boldsymbol{\lambda}}(s, T) e^{\boldsymbol{\mu}}(s, T) d s+\frac{i \mu^{(2)}}{S T} \int_{T_{0}}^{T}\left[\left.s \varepsilon_{\boldsymbol{\lambda}}(s, t) e^{\boldsymbol{\mu}}(s, t)\right|_{s=S_{0}} ^{S}\right] t d t \\
& -\frac{\mu^{(1)} \mu^{(2)}}{S T} \int_{S_{0}}^{S} \int_{T_{0}}^{T} s t \varepsilon_{\boldsymbol{\lambda}}(s, t) e^{\boldsymbol{\mu}}(s, t) d t d s .
\end{aligned}
$$

Put

$$
K(x)=i \frac{e^{-i x}-1}{x} \quad\left(=2 e^{-i x / 2} \frac{\sin (x / 2)}{x}\right)
$$


with $K(0)=1$ for continuity. Clearly we have $|K(x)|<1$ for $x \neq 0$. Using (*) and the computation of $I=I\left(S, T, S_{0}, T_{0}, \boldsymbol{\lambda}, \boldsymbol{\mu}\right)$ above, we get

$(* *) \quad \sigma_{f}\left(\boldsymbol{\lambda}+\boldsymbol{\mu}, S, T, S_{0}, T_{0}\right)$

$$
=\sigma_{f}(\boldsymbol{\lambda}) K\left(\mu^{(1)} S\right) K\left(\mu^{(2)} T\right)+R\left(S, T, S_{0}, T_{0}, \boldsymbol{\lambda}, \boldsymbol{\mu}\right),
$$

where

$$
\begin{aligned}
& R\left(S, T, S_{0}, T_{0}, \boldsymbol{\lambda}, \boldsymbol{\mu}\right)=\sigma_{f}(\boldsymbol{\lambda})\left(\frac{S_{0} T_{0}}{S T} K\left(\mu^{(1)} S_{0}\right) K\left(\mu^{(2)} T_{0}\right)\right. \\
& \left.-\frac{S_{0}}{S} K\left(\mu^{(1)} S_{0}\right) K\left(\mu^{(2)} T\right)-\frac{T_{0}}{T} K\left(\mu^{(1)} S\right) K\left(\mu^{(2)} T_{0}\right)\right)+I\left(S, T, S_{0}, T_{0}, \boldsymbol{\lambda}, \boldsymbol{\mu}\right) .
\end{aligned}
$$

Since the final expressions are compatible with approximations in the $L^{1}$ norm, $(* *)$ holds for arbitrary locally integrable $f$.

Write $I=I_{1}+I_{2}+I_{3}+I_{4}$ and assume that $\bar{\sigma}_{f}\left(\boldsymbol{\lambda}, S_{0}, T_{0}\right)<\infty$. Then $\lim _{S, T \rightarrow \infty} \varepsilon_{\boldsymbol{\lambda}}(S, T)=0$, and simple computations, using summability arguments, show that

$$
\lim _{S, T \rightarrow \infty} \sup _{\left\{\left|\mu^{(1)}\right| \leq 2 \pi / S,\left|\mu^{(2)}\right| \leq 2 \pi / T\right\}}\left|I_{j}\left(S, T, S_{0}, T_{0}, \boldsymbol{\lambda}, \boldsymbol{\mu}\right)\right|=0, \quad j=1,2,3,4,
$$

and finally, it follows that $\bar{\sigma}_{f}\left(\boldsymbol{\lambda}, S_{0}, T_{0}\right)<\infty$ implies

$$
\lim _{S, T \rightarrow \infty} \sup _{\left\{\left|\mu^{(1)}\right| \leq 2 \pi / S,\left|\mu^{(2)}\right| \leq 2 \pi / T\right\}}\left|R\left(S, T, S_{0}, T_{0}, \boldsymbol{\lambda}, \boldsymbol{\mu}\right)\right|=0 .
$$

Put

$$
d=\inf _{\max (|x|,|y|)=1}|K(x) K(y)-1|
$$

and recall that $d>0$. Given $\boldsymbol{\lambda}$ and $S_{0}, T_{0}$ such that $\bar{\sigma}_{f}\left(\boldsymbol{\lambda}, S_{0}, T_{0}\right)<\infty$, it follows from $(* *)$ and the uniform estimate for the remainder $R$ described above that for any preassigned $\delta>0$ we can find $\eta>0$ such that for any $\boldsymbol{\mu}$ with $0<\max \left(\left|\mu^{(1)}\right|,\left|\mu^{(2)}\right|\right)<\eta$ there exist $S \geq S_{0} / \delta, T \geq T_{0} / \delta$ satisfying $\max \left(\left|\mu^{(1)} S\right|,\left|\mu^{(2)} T\right|\right)=1$ and $\left|\sigma_{f}(\boldsymbol{\lambda}) K\left(\mu^{(1)} S\right) K\left(\mu^{(2)} T\right)-\sigma_{f}(\boldsymbol{\lambda}+\boldsymbol{\mu}, S, T)\right|$ $<\delta$. Then the definition of $d$ implies

$$
(\# \#) \quad\left|\sigma_{f}(\boldsymbol{\lambda})-\sigma_{f}\left(\boldsymbol{\lambda}+\boldsymbol{\mu}, S, T, S_{0}, T_{0}\right)\right|>\left|\sigma_{f}(\boldsymbol{\lambda})\right| d-\delta .
$$

Next, we claim that given any $\boldsymbol{\lambda} \in F$ with $\sigma_{f}(\boldsymbol{\lambda}) \neq 0$ and $S_{0}, T_{0}$ such that $\bar{\sigma}_{f}\left(\boldsymbol{\lambda}, S_{0}, T_{0}\right)<\infty$, we can find $\eta\left(=\eta\left(\boldsymbol{\lambda}, S_{0}, T_{0}\right)\right)>0$ such that for any $\boldsymbol{\mu}$ with $0<\max \left(\left|\mu^{(1)}\right|,\left|\mu^{(2)}\right|\right)<\eta$ there exist $S^{\prime} \geq S_{0}, T^{\prime} \geq T_{0}$ satisfying

$$
\left|\sigma_{f}(\boldsymbol{\lambda})-\sigma_{f}\left(\boldsymbol{\lambda}+\boldsymbol{\mu}, S^{\prime}, T^{\prime}\right)\right|>\left|\sigma_{f}(\boldsymbol{\lambda})\right| \frac{d}{2} .
$$

For this, we choose $\delta>0$ so that $\delta\left(1+3\left|\sigma_{f}(\boldsymbol{\lambda})\right|(1+d / 2)\right)<(d / 2)\left|\sigma_{f}(\boldsymbol{\lambda})\right|$, and a corresponding $\eta>0$ leading to $(\# \#)$. Then, if $S, T$ satisfy $(\# \#)$, we want to show that at least one $\left(S^{\prime}, T^{\prime}\right) \in\left\{(S, T),\left(S_{0}, T\right),\left(S, T_{0}\right),\left(S_{0}, T_{0}\right)\right\}$ must 
satisfy $(* * *)$. We argue by contradiction, i.e., we assume that the converse of inequality $(* * *)$ holds for these four points. Using (\#), this implies

$$
\begin{aligned}
&\left|\left(1-\frac{S_{0}}{S}\right)\left(1-\frac{T_{0}}{T}\right) \sigma_{f}(\boldsymbol{\lambda})-\sigma_{f}\left(\boldsymbol{\lambda}+\boldsymbol{\mu}, S, T, S_{0}, T_{0}\right)\right| \\
&=\mid\left(\sigma_{f}(\boldsymbol{\lambda})-\sigma_{f}(\boldsymbol{\lambda}+\boldsymbol{\mu}, S, T)\right)-\frac{S_{0}}{S}\left(\sigma_{f}(\boldsymbol{\lambda})-\sigma_{f}\left(\boldsymbol{\lambda}+\boldsymbol{\mu}, S_{0}, T\right)\right) \\
& \quad-\frac{T_{0}}{T}\left(\sigma_{f}(\boldsymbol{\lambda})-\sigma_{f}\left(\boldsymbol{\lambda}+\boldsymbol{\mu}, S, T_{0}\right)\right)+\frac{S_{0} T_{0}}{S T}\left(\sigma_{f}(\boldsymbol{\lambda})-\sigma_{f}\left(\boldsymbol{\lambda}+\boldsymbol{\mu}, S_{0}, T_{0}\right)\right) \mid \\
& \leq\left(1+\frac{S_{0}}{S}\right)\left(1+\frac{T_{0}}{T}\right)\left|\sigma_{f}(\boldsymbol{\lambda})\right| \frac{d}{2} .
\end{aligned}
$$

Combining this with the lower bounds for $S, T$ and with (\#\#), we arrive at

$$
\begin{aligned}
\left|\sigma_{f}(\boldsymbol{\lambda})\right| d-\delta & <\left|\sigma_{f}(\boldsymbol{\lambda})-\sigma_{f}\left(\boldsymbol{\lambda}+\boldsymbol{\mu}, S, T, S_{0}, T_{0}\right)\right| \\
& <3 \delta\left|\sigma_{f}(\boldsymbol{\lambda})\right|+(1+3 \delta)\left|\sigma_{f}(\boldsymbol{\lambda})\right| \frac{d}{2}=\left|\sigma_{f}(\boldsymbol{\lambda})\right|\left(3 \delta+(1+3 \delta) \frac{d}{2}\right) ;
\end{aligned}
$$

but this contradicts our choice of $\delta$, proving $(* * *)$.

Now for $\varepsilon>0$ given, we show that $F_{\varepsilon}$ is scattered. Assume the contrary; then $F_{\varepsilon}$ contains a (necessarily infinite) subset $A$ which is dense in itself (i.e., every point of $A$ is an accumulation point of $A$ ). We will construct inductively a decreasing sequence $\left(B_{j}\right)$ of open balls in the plane which intersect $A$, and two non-decreasing sequences $\left(S_{j}\right)$ and $\left(T_{j}\right)$ tending to infinity, with the property that

$$
\left|\sigma_{f}\left(\boldsymbol{\lambda}, S_{2 j}, T_{2 j}\right)-\sigma_{f}\left(\boldsymbol{\lambda}, S_{2 j+1}, T_{2 j+1}\right)\right|>\varepsilon \frac{d}{4} \quad \text { for all } \boldsymbol{\lambda} \in B_{j}, j \geq 1 .
$$

Let $B_{0}$ be the whole plane and choose $S_{1}, T_{1}$ arbitrarily. For $j>0$, assume we have already defined $B_{j-1}, S_{2 j-1}, T_{2 j-1}$. Take an arbitrary $\boldsymbol{\lambda}_{j} \in B_{j-1} \cap A$ and choose $S_{2 j}>S_{2 j-1}+1, T_{2 j}>T_{2 j-1}+1$ satisfying $\bar{\sigma}_{f}\left(\boldsymbol{\lambda}_{j}, S_{2 j}, T_{2 j}\right)<\infty$ and $\left|\sigma_{f}\left(\boldsymbol{\lambda}_{j}\right)-\sigma_{f}\left(\boldsymbol{\lambda}_{j}, S_{2 j}, T_{2 j}\right)\right|<\varepsilon d / 8$. Now consider an open ball $B^{\prime}$ around $\boldsymbol{\lambda}_{j}$ of radius $\eta>0$ such that $B^{\prime} \subseteq B_{j-1}, \eta \leq$ $\eta\left(\boldsymbol{\lambda}_{j}, S_{2 j}, T_{2 j}\right)$ and $\left|\sigma_{f}\left(\boldsymbol{\lambda}_{j}\right)-\sigma_{f}\left(\boldsymbol{\lambda}, S_{2 j}, T_{2 j}\right)\right|<\varepsilon d / 8$ for all $\boldsymbol{\lambda} \in B^{\prime}$ (observe that $\sigma_{f}(\boldsymbol{\lambda}, S, T)$ depends continuously on $\left.\boldsymbol{\lambda}\right)$. Since $A$ is dense in itself, there exists $\boldsymbol{\mu} \neq \mathbf{0}$ such that $\boldsymbol{\lambda}^{\prime}=\boldsymbol{\lambda}_{j}+\boldsymbol{\mu} \in B^{\prime} \cap A$. Then (with $\boldsymbol{\lambda}=\boldsymbol{\lambda}_{j}, S_{0}=S_{2 j}$, $\left.T_{0}=T_{2 j}\right)$ choose $S_{2 j+1}\left(=S^{\prime}\right), T_{2 j+1}\left(=T^{\prime}\right)$ satisfying $(* * *)$. Finally, take for $B_{j}$ an open ball around $\boldsymbol{\lambda}^{\prime}$ such that $B_{j} \subseteq B^{\prime}$ and $\mid \sigma_{f}\left(\boldsymbol{\lambda}, S_{2 j+1}, T_{2 j+1}\right)-$ $\sigma_{f}\left(\boldsymbol{\lambda}^{\prime}, S_{2 j+1}, T_{2 j+1}\right) \mid<\varepsilon d / 8$ for all $\boldsymbol{\lambda} \in B_{j}$. Then it is easy to see that $B_{j}, S_{2 j}, T_{2 j}, S_{2 j+1}, T_{2 j+1}$ have the properties stated above.

Take $\boldsymbol{\lambda} \in \bigcap_{j \geq 1} \bar{B}_{j}$. Our construction implies that $\lim _{j \rightarrow \infty} \sigma_{f}\left(\boldsymbol{\lambda}, S_{j}, T_{j}\right)$ does not exist, giving a contradiction. Hence $F_{\varepsilon}$ is scattered and the theorem is proved. 
COROLlary 4.4. Let $f(s, t)$ be a locally integrable function on $[0, \infty) \times$ $[0, \infty)$. Then the set of $\boldsymbol{\lambda} \in \mathbb{R}^{2}$ where the limit $\sigma_{f}(\boldsymbol{\lambda})$ exists and has a value different from zero is at most countable.

Proof. This follows from Theorem 4.3 in the same way as in [8, Théorème 2].

REMARKS. 1. For a fixed value of $\boldsymbol{\lambda}$, say $\boldsymbol{\lambda}=\mathbf{0}$, it is easy to give examples of functions $f$ for which $\sigma_{f}(\mathbf{0})$ exists and $\bar{\sigma}_{f}(\mathbf{0}, S, T)=\infty$ for some $S, T$. Take, e.g., $f(s, t)=n$ for $(s, t) \in[n-1, n) \times[0,1), f(s, t)=-n$ for $(s, t) \in$ $[n-1, n) \times[1,2), f(s, t)=0$ otherwise. Then $\bar{\sigma}_{f}(\mathbf{0}, S, 1)=\infty$ for all $S$ and $\lim _{S, T \rightarrow \infty} \sigma_{f}\left(\boldsymbol{\lambda}, S, T, S_{0}, 1\right)$ exists for no $S_{0}$. But of course, in this example $\sigma_{f}\left(0, \lambda^{(2)}\right)$ does not exist for $\lambda^{(2)} \neq 0,2 \pi, \ldots$

In the discrete case, one can show in a similar way to Proposition 5.1 below that if $f$ is Hartman almost periodic on $\mathbb{N}^{2}$ (with $A_{N M}=[0, N] \times$ $[0, M], N, M>0)$, then $\bar{\sigma}_{f}(\boldsymbol{\lambda}, N, M)<\infty$ for all $\boldsymbol{\lambda}, N, M$.

On the other hand, on $[0, \infty)^{2}$ (the setting of Theorem 4.3), one can use constructions as in Remark 1 of Section 5 to find Hartman almost periodic functions $f$ for which $\bar{\sigma}_{f}(\boldsymbol{\lambda}, S, 1)=\infty$ for all $\boldsymbol{\lambda}, S$ and $\bar{\sigma}_{f}(\boldsymbol{\lambda}, S, T)<\infty$ for all $\boldsymbol{\lambda}, S$ when $T \geq 2$.

2. The proof gets easier when $\bar{\sigma}_{f}(\boldsymbol{\lambda}, S, T)<\infty$ for all $\boldsymbol{\lambda}, S, T$ (and one can follow more closely the pattern of [8]). This holds in particular if $f \in \mathcal{M}_{1}$. More generally, the weaker condition

$$
\sup _{S, T>0} \sup _{\lambda^{(1)}, \lambda^{(2)} \in \mathbb{R}}\left|\frac{1}{S T} \int_{0}^{S} \int_{0}^{T} f(s, t) e^{-i\left(\lambda^{(1)} s+\lambda^{(2)} t\right)} d s d t\right|<\infty
$$

is sufficient (this is a special case of the condition in Berend et al. [3, Theorem 4.2]).

3. With some further arguments, the same conclusions as in Theorem 4.3 and Corollary 4.4 can be shown for $A_{S T}=[-S, S] \times[-T, T],(S, T) \in \mathbb{R}^{+} \times \mathbb{R}^{+}$ (even in the one-dimensional case, the extension of Kahane's theorem to symmetric averages over $[-T, T]$ or $[-N, N]$ requires some additional techniques).

4. Another notion of almost periodicity in the two-dimensional case was considered by Hartman in [6, p. 350], providing also a generalization of Kahane's theorem $\left(\left[6\right.\right.$, Satz 5]). It is based on iterated $\operatorname{limits} \lim _{S \rightarrow \infty} \lim _{T \rightarrow \infty}$ and does not fit into the general scheme of our definition.

Corollary 4.5. Let $\left(a_{n m}\right)$ be a (double) sequence of complex numbers. For $\boldsymbol{\lambda}$ in $[-\pi, \pi]^{2}\left(\boldsymbol{\lambda}=\left(\lambda^{(1)}, \lambda^{(2)}\right)\right)$ consider the unrestricted limit

$$
\sigma_{a}(\boldsymbol{\lambda}):=\lim _{N, M \rightarrow \infty} \frac{1}{N M} \sum_{n=1}^{N} \sum_{m=1}^{M} a_{n m} e^{-i\left(\lambda^{(1)} n+\lambda^{(2)} m\right)} .
$$


Then the set of $\boldsymbol{\lambda} \in[-\pi, \pi]^{2}$ where the limit $\sigma_{a}(\boldsymbol{\lambda})$ exists and has a value different from zero is at most countable. Furthermore, if $F$ is a closed subset of $[-\pi, \pi]^{2}$ and $\sigma_{a}(\boldsymbol{\lambda})$ exists for each $\boldsymbol{\lambda} \in F$, then given $\varepsilon>0$, the set $F_{\varepsilon}:=\left\{\boldsymbol{\lambda} \in F:\left|\sigma_{a}(\boldsymbol{\lambda})\right| \geq \varepsilon\right\}$ is scattered.

Proof. Again, we write $e^{\boldsymbol{\lambda}}(s, t)=e^{-i\left(\lambda^{(1)} s+\lambda^{(2)} t\right)}$. Take some $\boldsymbol{\lambda}$ for which $\sigma_{a}(\boldsymbol{\lambda})$ exists. Then it follows from the discrete counterpart of (\#) that

$$
\begin{aligned}
\lim _{N, M \rightarrow \infty} \frac{a_{N M}}{N M}=0, \quad \lim _{N, M \rightarrow \infty} \frac{1}{N M} \sum_{m=1}^{M} a_{N m} e^{\boldsymbol{\lambda}}(N, m) & =0, \\
\lim _{N, M \rightarrow \infty} \frac{1}{N M} \sum_{n=1}^{N} a_{n M} e^{\boldsymbol{\lambda}}(n, M) & =0 .
\end{aligned}
$$

Denote by $[x]$ the greatest integer not exceeding $x$ and note that $[x] / x \rightarrow 1$ as $x \rightarrow \infty$.

Put $f(s, t)=a_{n m}$ for $(s, t) \in[n-1, n) \times[m-1, m)$, extending $\left(a_{n m}\right)$ to a function $f$ on $[0, \infty)^{2}$. Now, for $\lambda^{(1)}, \lambda^{(2)} \neq 0$, easy computations show that

$$
\begin{aligned}
& \int_{0}^{N} \int_{0}^{M} f(s, t) e^{\boldsymbol{\lambda}}(s, t) d s d t=\frac{\left(1-e^{i \lambda^{(1)}}\right)\left(1-e^{i \lambda^{(2)}}\right)}{-\lambda^{(1)} \lambda^{(2)}} \sum_{n=1}^{N} \sum_{m=1}^{M} a_{n m} e^{\boldsymbol{\lambda}}(n, m), \\
& \int_{0}^{S} \int_{0}^{T} f(s, t) e^{\boldsymbol{\lambda}}(s, t) d s d t=\int_{0}^{[S]} \int_{0}^{[T]} f(s, t) e^{\boldsymbol{\lambda}}(s, t) d s d t+o(S T) .
\end{aligned}
$$

It follows that $\sigma_{f}(\boldsymbol{\lambda})$ exists if and only if $\sigma_{a}(\boldsymbol{\lambda})$ exists and that

$$
\sigma_{f}(\boldsymbol{\lambda})=\frac{\left(1-e^{i \lambda^{(1)}}\right)\left(1-e^{i \lambda^{(2)}}\right)}{-\lambda^{(1)} \lambda^{(2)}} \sigma_{a}(\boldsymbol{\lambda}) .
$$

The case where $\lambda^{(1)}$ or $\lambda^{(2)}$ are equal to zero can be handled in a similar way. Now we can apply Theorem 4.3 and Corollary 4.4 .

REMARKs. 5. A further generalization of Theorem 4.3 is to replace $f$ by a finite measure $\varphi$ on the ring of bounded Borel sets in $[0, \infty) \times[0, \infty)$ which is $\sigma$-additive on the subsets of $[0, N]^{2}$ for all $N$. Then formula $(* *)$ still holds (by w*-approximation) if $\int_{S_{0}}^{S} \int_{T_{0}}^{T}$ is interpreted as the integral over the closed rectangle $\left[S_{0}, S\right] \times\left[T_{0}, T\right]$. This also contains Corollary 4.5 above.

6. If $G$ is a compactly generated, locally compact, abelian group, then a classical structure theorem ([7, Theorem II.9.8]) gives an isomorphism $G \cong \mathbb{R}^{a} \times \mathbb{Z}^{b} \times K$, where $a, b \geq 0$ and $K$ is compact. This can be used to define a family $\left(A_{i}\right)$ (indexed by $\left.\left(\mathbb{R}^{+}\right)^{a} \times \mathbb{N}^{b}\right)$ so that the conclusions of Theorem 4.3 and Corollary 4.4 hold for the corresponding class of Hartman almost periodic functions (or measures). On the other hand, the following 
example shows that this does not hold for other families $\left(A_{i}\right)$ that are common when defining limits in multi-dimensional harmonic analysis (compare Example 2.3(iv)).

EXAmple 4.6. For $G=\mathbb{Z}^{2}, A_{N}=[1, N]^{2}(N=1,2, \ldots)$, there exists a (double) sequence $a=\left(a_{n m}\right) \in \mathcal{M}_{1}$ for which the limit

$$
\sigma_{a}\left(\lambda^{(1)}, \lambda^{(2)}\right):=\lim _{N \rightarrow \infty} \frac{1}{N^{2}} \sum_{n=1}^{N} \sum_{m=1}^{N} a_{n m} e^{-i\left(\lambda^{(1)} n+\lambda^{(2)} m\right)}
$$

exists for every $\left(\lambda^{(1)}, \lambda^{(2)}\right) \in[-\pi, \pi]^{2}$ (thus $a$ is Hartman almost periodic), but $\left|\sigma_{a}\left(0, \lambda^{(2)}\right)\right|=1 / 2$ for every $\lambda^{(2)} \in[-\pi, \pi]$.

Define $a_{n m}$ as follows: put $a_{n 1}=n$ for any $n \geq 1$, otherwise put $a_{n m}=0$. Clearly, we have

$$
\sigma_{a}\left(0, \lambda^{(2)}\right)=\lim _{N \rightarrow \infty} \frac{e^{-i \lambda^{(2)}}}{N^{2}} \sum_{n=1}^{N} n=\frac{1}{2} .
$$

On the other hand, $D_{n}(\mu)=\sum_{k=1}^{n} e^{i \mu k}$ satisfies $\left|D_{n}(\mu)\right| \leq 4 /|\mu|$ for $0<$ $|\mu| \leq \pi$, hence Abel's summation by parts gives

$$
\left|\sum_{n=1}^{N} n e^{i \mu n}\right|=\left|N D_{N}(\mu)-\sum_{n=1}^{N-1} D_{n}(\mu)\right| \leq \frac{8 N}{|\mu|} .
$$

Thus $\sigma_{a}\left(\lambda^{(1)}, \lambda^{(2)}\right)=0$ if $\lambda^{(1)} \neq 0$.

5. Some final observations. We discuss some further results in the one-dimensional case, concerning subsequential convergence with respect to various families of subsets $\left(A_{i}\right)$.

Proposition 5.1. Let $\left(a_{n}\right)$ be a sequence of complex numbers. Let $\left(N_{k}\right)$ be a subsequence of $\mathbb{N}$ with bounded gaps, i.e.,

and assume that the limit

$$
M=\limsup _{k \geq 1}\left(N_{k+1}-N_{k}\right)<\infty,
$$

$$
\sigma_{a}(\lambda):=\lim _{k \rightarrow \infty} \frac{1}{N_{k}} \sum_{j=1}^{N_{k}} a_{j} e^{-i \lambda j}
$$

exists for every $\lambda \in F$, where $F \subseteq[0,2 \pi]$ has at least $M$ elements. Then the limit $\sigma_{a}(\lambda)=\lim _{N \rightarrow \infty} N^{-1} \sum_{j=1}^{N} a_{j} e^{-i \lambda j}$ exists for all $\lambda \in F$. Therefore, $\sigma_{a}(\lambda)$ has the same properties as in Theorem 4.3 and Corollary 4.5.

Proof. Let $\lambda_{1}, \ldots, \lambda_{M} \in F$ be distinct elements. By the existence of the limit at these points, and since $N_{k+1} / N_{k} \rightarrow 1$, we have 
$\frac{1}{N_{k+1}} \sum_{j=1}^{N_{k+1}} a_{j} e^{-i \lambda_{v} j}-\frac{1}{N_{k+1}} \sum_{j=1}^{N_{k}} a_{j} e^{-i \lambda_{v} j}=$
$\frac{1}{N_{k+1}} e^{-i \lambda_{v}\left(N_{k}+1\right)}\left(a_{N_{k}+1}+a_{N_{k}+2} e^{-i \lambda_{v}}+\cdots+a_{N_{k+1}} e^{-i \lambda_{v}\left(N_{k+1}-N_{k}-1\right)}\right) \rightarrow{ }_{k} 0$ for $v=1,2, \ldots, N_{k+1}-N_{k}$ (which is not greater than $M$ if $k \geq k_{0}$ is large enough). Put $M_{k}=N_{k+1}-N_{k}-1$; writing the above relations in matrix form, we have

$$
\frac{1}{N_{k+1}}\left(\begin{array}{cccc}
1 & e^{-i \lambda_{1}} & \cdots & e^{-i \lambda_{1} M_{k}} \\
1 & e^{-i \lambda_{2}} & \cdots & e^{-i \lambda_{2} M_{k}} \\
\vdots & \vdots & \cdots & \vdots \\
1 & e^{-i \lambda_{M_{k}}} & \cdots & e^{-i \lambda_{M_{k} M_{k}}} \\
1 & e^{-i \lambda_{M_{k}+1}} & \cdots & e^{-i \lambda_{M_{k}+1} M_{k}}
\end{array}\right)\left(\begin{array}{c}
a_{N_{k}+1} \\
a_{N_{k}+2} \\
\vdots \\
a_{N_{k+1}-1} \\
a_{N_{k+1}}
\end{array}\right) \rightarrow 0 \quad \text { as } k \rightarrow \infty
$$

Denote by $\left(V_{k}\right)$ the sequence of matrices arising above. $V_{k}$ is a Vandermonde matrix of order at most $M$, for every $k \geq k_{0}$. Since $\lambda_{1}, \ldots, \lambda_{M}$ are all distinct, $V_{k}$ is invertible, hence $\sup _{k \geq k_{0}}\left\|V_{k}^{-1}\right\|<\infty$. We have

$$
\frac{1}{N_{k+1}}\left\|\left(a_{N_{k}+1}, \ldots, a_{N_{k+1}}\right)^{\prime}\right\| \leq\left\|V_{k}^{-1}\right\| \cdot \frac{1}{N_{k+1}}\left\|V_{k}\left(a_{N_{k}+1}, \cdots, a_{N_{k+1}}\right)^{\prime}\right\| \rightarrow 0
$$

(where' denotes transpose). This yields

$$
\max _{N_{k}<j \leq N_{k+1}} \frac{\left|a_{j}\right|}{N_{k+1}} \rightarrow 0 .
$$

Now, let $N_{k}<n \leq N_{k+1}$. Then

$$
\begin{aligned}
& \frac{1}{n}\left|\sum_{j=1}^{n} a_{j} e^{-i \lambda j}-\sigma_{a}(\lambda)\right| \\
& \quad \leq \frac{1}{N_{k}}\left|\sum_{j=1}^{N_{k}} a_{j} e^{-i \lambda j}-\sigma_{a}(\lambda)\right|+\left(N_{k+1}-N_{k}\right) \frac{1}{N_{k}} \max _{N_{k}<j \leq N_{k+1}}\left|a_{j}\right| .
\end{aligned}
$$

Combined, this gives

$$
\lim _{n \rightarrow \infty} \frac{1}{n} \sum_{j=1}^{n} a_{j} e^{-i \lambda j}=\sigma_{a}(\lambda),
$$

and we may apply the original theorem of Kahane.

REMARKS. 1. In the continuous one-dimensional case, the statement corresponding to Proposition 5.1 is false. Take any strictly increasing sequence $\left(T_{n}\right)$ of real numbers tending to infinity, and consider the real intervals $A_{n}=\left[0, T_{n}\right]$. Given any continuous function $g: \mathbb{R} \rightarrow \mathbb{C}$, one can construct a locally integrable function $f$ such that 


$$
\lim _{n \rightarrow \infty} \frac{1}{T_{n}} \int_{0}^{T_{n}} f(t) e^{-i \lambda t} d t=g(\lambda) \quad \text { for all } \lambda \in \mathbb{R} .
$$

This can be done as follows: let $R_{a, b} h$ denote the restriction of a function $h$ to the interval $[a, b]$. By an easy duality argument, one can see that for any finite numbers $a<b, c<d$ the set $\left\{R_{a, b} \widehat{f}: f \in L_{1}([c, d])\right\}$ is dense in the space of continuous functions on $[a, b]$ with respect to $\|\cdot\|_{\infty}$. Indeed: otherwise, by the Hahn-Banach theorem, there exists a non-zero measure $\mu$ on $[a, b]$ such that $\int \widehat{f} d \mu=0$ for all $f \in L_{1}([c, d])$. By the inversion theorem this would imply that the Fourier-Stieltjes transform $\widehat{\mu}$ vanishes on $[-d,-c]$ - this is impossible since $\widehat{\mu}$ is the restriction of a non-zero entire analytic function.

Then, by induction, assuming that $f_{j}$ have been defined for $1 \leq j<n$, we can find $f_{n} \in L_{1}\left(\left[T_{n-1}, T_{n}\right]\right)$ such that

$$
\left\|R_{-T_{n}, T_{n}}\left(g-\frac{1}{T_{n}} \sum_{j=1}^{n} \widehat{f}_{j}\right)\right\|_{\infty}<\frac{1}{n} \quad(n=1,2, \ldots) .
$$

Clearly, we put $f_{n}(t)=0$ for $t \notin\left[T_{n-1}, T_{n}\right]$, and then $f=\sum_{j=1}^{\infty} f_{j}$ will do the job.

Such a behaviour cannot occur for functions $f \in \mathcal{M}_{1}$ : see Remark 3 below.

2. In [8, Remarque 1], an example is given of a function $f$ on $\mathbb{R}$ for which $\sigma_{f}(\lambda)=-i / 2$ for infinitely many $\lambda$ (with $A_{t}=[0, t]$ for $t \in \mathbb{R}, t>0$ ). But with the definition of [8], $\sigma_{f}(0)$ does not exist, hence $f$ is not Hartman almost periodic. As a remedy, this can be modified as follows. Given a strictly decreasing sequence $\left(\varepsilon_{n}\right)$ with $\varepsilon_{n}>0$ and $\sum \varepsilon_{n}<\infty$, choose an increasing sequence $\left(T_{n}\right), T_{0}=0$, such that

$$
\frac{1}{T_{N}} \sum_{n=1}^{N} \frac{1}{\varepsilon_{n}} \rightarrow 0 \quad \text { as } N \rightarrow \infty
$$

and put $f(t)=\sum_{n=1}^{N} \sin \left(\varepsilon_{n} t\right)$ for $T_{N-1} \leq t<T_{N}, f(t)=0$ for $t<0$. Then $f$ has the properties as claimed in [8], in particular $f$ is Hartman almost periodic and $\sigma_{f}\left( \pm \varepsilon_{n}\right)=-i / 2$ for all $n$. In this example the stronger properties of Corollary 3.2 do not hold.

More generally, one can consider $f(t)=\sum_{n=1}^{N} \alpha_{n} \sin \left(\varepsilon_{n} t\right)$ as above, assuming $\sum\left|\alpha_{n} \varepsilon_{n}\right|<\infty$ and $\left(T_{n}\right)$ is chosen so that

$$
\frac{1}{T_{N}} \sum_{n=1}^{N}\left|\frac{\alpha_{n}}{\varepsilon_{n}}\right| \rightarrow 0 \quad \text { as } N \rightarrow \infty \text {. }
$$

Again $f$ is Hartman almost periodic and $\sigma_{f}\left( \pm \varepsilon_{n}\right)=-i \alpha_{n} / 2$ for all $n$. Thus $\sigma_{f}(\lambda)$ can be unbounded. 
3. If $\left(N_{k}\right)$ is an increasing sequence of real (or integer) numbers for which $N_{k+1} / N_{k} \rightarrow 1$, and a function $f$ on $\mathbb{R}$ (or $\mathbb{Z}$ ) is in $\mathcal{M}_{1}$, then it can be shown by similar techniques to those of Section 4 that $\sigma_{f}(\lambda)$ (defined with respect to $\left.A_{k}=\left[0, N_{k}\right], k=1,2, \ldots\right)$ has the same properties as described in Theorem 4.3 and Corollary 4.5. But in general, the existence of the limit for $A_{k}=\left[0, N_{k}\right]$ no longer implies the existence of the limit for the "full" family $[0, t], t>0$.

4. Let $\left(N_{k}\right)$ be an increasing sequence for which $N_{k+1} / N_{k} \rightarrow \infty$ and consider again limits defined with respect to $A_{k}=\left[0, N_{k}\right](k=1,2, \ldots)$. Then, using Riesz products, one can define a function $f$ on $\mathbb{R}$ such that $f \geq 0, f \in \mathcal{M}_{1}, f$ is Hartman almost periodic and $\sigma_{f}(\lambda)=1$ for uncountably many $\lambda$.

5. If $G$ is any locally compact abelian group, and $f \in \mathcal{M}_{1}$ is Hartman almost periodic, then similar to Theorem 3.1, there exists a measure $\varphi$ on $G^{*}$ such that $\sigma_{f}=\widehat{\varphi}$ (thus $\sigma_{f}$ is a linear combination of positive definite functions). Furthermore, if $\mu\left(A_{i}\right) \rightarrow \infty$, it is easy to see that $\sigma_{f}(\chi)=0$ almost everywhere (with respect to Haar measure on $\widehat{G}$; equivalently, $\varphi$ vanishes on $G$ ). We restrict to sequences $\left(A_{k}\right)$ with $\mu\left(A_{k}\right) \rightarrow \infty$ (or to families $\left(A_{i}\right)$ having a countable cofinal subfamily with this property). If $\varphi$ is any measure on $G^{*} \backslash G$, an abstract characterization for the existence of a family $\left(A_{k}\right)$ such that $\widehat{\varphi}=\sigma_{f}$ for some $f \in \mathcal{M}_{1}$ are the conditions a), b) of $\left[14\right.$, Theorem 1]. Clearly, $\sigma_{f}$ has to be of first Baire class. Since $\sigma_{f}(\chi)=0$ at every point of continuity, it follows easily that $\left\{\chi \in \widehat{G}:\left|\sigma_{f}(\chi)\right| \geq \varepsilon\right\}$ has to be a nowhere dense set of measure zero for every $\varepsilon>0$ (this gives a necessary condition for any sequence $\left(A_{k}\right)$ as above and any Hartman almost periodic $f \in \mathcal{M}_{1}$ ).

We return to the case $G=\mathbb{R}$ (similarly for $G=\mathbb{Z}$ ). Fixing a particular family $\left(A_{i}\right)$ imposes further restrictions on the measures $\varphi$ (respectively functions $\widehat{\varphi}$ ) that can appear, e.g., in Kahane's theorem for the "full" family $A_{T}=[0, T]$. Even in that case one can construct (similarly to Remark 3) a Hartman almost periodic $f \in \mathcal{M}_{1}$ for which $\left\{\lambda \in \mathbb{R}:\left|\sigma_{f}(\lambda)\right| \geq \varepsilon\right\}$ is infinite for some $\varepsilon>0$. Hence, in this example $\varphi \notin L_{1}\left(G^{*}, \mu^{*}\right)$, i.e., the analogue of Theorem 3.1 does not hold. As a further property in this example, $\left\{\lambda \in \mathbb{R}:\left|\sigma_{f}(\lambda)\right|>0\right\}$ need not be scattered. Remark 3 (and also Example 4.6) shows that different families $\left(A_{i}\right)$ can give less restricted classes of measures $\varphi$.

\section{References}

[1] M. A. Akcoglu and A. del Junco, Convergence of averages of point transformations, Proc. Amer. Math. Soc. 49 (1975), 265-266. 
[2] A. Bellow and V. Losert, The weighted pointwise ergodic theorem and the individual ergodic theorem along subsequences, Trans. Amer. Math. Soc. 288 (1985), 307-345.

[3] D. Berend, M. Lin, J. Rosenblatt and A. Tempelman, Modulated and subsequential ergodic theorems in Hilbert and Banach spaces, Ergodic Theory Dynam. Systems 22 (2002), 1653-1665.

[4] J.-P. Bertrandias, J. Couot, J. Dhombres, M. Mendès France, Pham Phu Hien and Kh. Vo-Khac, Espaces de Marcinkiewicz: corrélations, mesures, systèmes dynamiques, with an introduction by J. Bass, Masson, Paris, 1987.

[5] D. Çömez, M. Lin and J. Olsen, Weighted ergodic theorems for mean ergodic $L_{1}$ contractions, Trans. Amer. Math. Soc. 350 (1998), 101-117.

[6] S. Hartman, Verallgemeinerte harmonische Analysis, Ann. Polon. Math. 16 (1965), 341-352.

[7] E. Hewitt and K. A. Ross, Abstract Harmonic Analysis, Vol. I, Grundlehren Math. Wiss. 115, Springer, Berlin, 1963.

[8] J.-P. Kahane, Sur les coefficients de Fourier-Bohr, Studia Math. 21 (1961), 103-106.

[9] —, Sur les fonctions presque-périodiques généralisées dont le spectre est vide, ibid. 21 (1962), 231-236.

[10] - Some Random Series of Functions, 2nd ed., Cambridge Univ. Press, Cambridge, 1985.

[11] M. Lin and J. Olsen, Besicovitch functions and weighted ergodic theorems for LCA group actions, in: Convergence in Ergodic Theory and Probability (Columbus, $\mathrm{OH}$, 1993), Ohio State Univ. Math. Res. Inst. Publ. 5, de Gruyter, Berlin, 1996, 277-289.

[12] M. Lin, J. Olsen and A. Tempelman, On modulated ergodic theorems for DunfordSchwartz operators, Illinois J. Math. 43 (1999), 542-567.

[13] M. Lin and A. Tempelman, Averaging sequences and modulated ergodic theorems for weakly almost periodic group representations, J. Anal. Math. 77 (1999), 237-268.

[14] V. Losert, On the existence of uniformly distributed sequences in compact topological spaces. II, Monatsh. Math. 87 (1979), 247-260.

[15] J. Marcinkiewicz, Une remarque sur les espaces de M. Besikowitch, C. R. Acad. Sci. Paris 208 (1939), 157-159.

[16] J.-P. Pier, Amenable Locally Compact Groups, Wiley, New York, 1984.

[17] C. Ryll-Nardzewski, Topics in ergodic theory, in: Lecture Notes in Math. 472, Springer, Berlin, 1975, 131-156.

[18] A. A. Tempelman, An ergodic theorem for amplitude modulated homogeneous random fields, Litovsk. Math. Sb. 14 (1974), no. 4, 221-229 (in Russian); English transl.: Lithuanian Math. Transl. 14 (1974), 698-704.

[19] K. Urbanik, Fourier analysis in Marcinkiewicz spaces, Studia Math. 21 (1961), 93-102.

[20] N. Wiener and A. Wintner, Harmonic analysis and ergodic theory, Amer. J. Math. 63 (1941), 415-426.

Institute of Mathematics

Hebrew University

Givat Ram

Jerusalem 91904, Israel

E-mail: gc@math.huji.ac.il
Fakultät für Mathematik

Universität Wien

Nordbergstr. 15

A-1090 Wien, Austria

E-mail: viktor.losert@univie.ac.at 